\title{
All-Optical Multicast Routing in Sparse Splitting WDM Networks
}

\author{
Cheng-Yu Hsieh and Wanjiun Liao, Senior Member, IEEE
}

\begin{abstract}
This paper studies all-optical multicast routing in wavelength-routed optical networks with sparse light splitting. In a sparse splitting network, only a small percentage of nodes is capable of light splitting, i.e., multicast capable, and most of the nodes are multicast incapable. The typical approach to this problem is combining an existing Steiner tree heuristics with some rerouting procedure to refine the trees. Therefore, the cost in terms of the total number of wavelengths used for all tree links (referred to as wavelength channel cost) is very high. In this paper, we propose a new mechanism that constructs light-trees for sparse splitting optical networks without additional rerouting. We design two efficient schemes to build a light-tree for any given multicast session. We then extend our mechanism to support dynamic group membership. The simulation results show that our mechanism can build light-trees with the least wavelength channel cost and with the smallest number of wavelengths used per link.
\end{abstract}

Index Terms-All optical multicast routing, WDM, light-tree, sparse light splitting.

\section{INTRODUCTION}

$\mathbf{W}$ AVELENGTH-division multiplexing (WDM) in optical fiber networks is an important technology to meet the ever-increasing bandwidth demands of network users. In a wavelength-routed WDM network, communications between end users are via all-optical WDM channels referred to as lightpaths. Data transmitted through a lightpath need no electronic processing at intermediate nodes. Therefore, optical bandwidths in WDM networks can be used more efficiently.

Multicast is an efficient way of one-to-many or many-tomany communications. A multicast session typically consist of a source and a set of receivers. To avoid sending a separate copy to each of the receivers in the group, a multicast session is typically implemented with a multicast tree, in which the root is located at the source and the branches span all the receivers. The way of how a multicast tree grows is determined by the multicast routing protocol. To support multicast at the WDM layer, the concept of the light-tree was first introduced in [1]. A light-tree is a point-to-multipoint extension of a light-path. For each light-tree, the source node is the only node that is associated with a transmitter, and intermediate nodes are equipped with light (optical) splitters. A node with splitting capability can forward an incoming signal to multiple output channels without transmitters, and thus is said to be

Manuscript received March 24, 2005; revised March 18, 2007. This work was supported by National Science Council (NSC), Taiwan, under a Center Excellence Grant NSC95-2752-E-002-006-PAE, and under Grant Number NSC95-2221-E-002-066.

C.-Y. Hsieh and W. Liao are with the Department of Electrical Engineering and the Graduate Institute of Communication Engineering, National Taiwan University, Taipei, Taiwan (corresponding author email: wjliao@ntu.edu.tw). Digital Object Identifier 10.1109/JSAC-OCN.2007.021305.
Multicast-Capable (MC). An MC node, however, is expensive to implement due to the complexity of fabrication and the large number of amplifiers required. The concept of sparsesplitting was first introduced in [2]. With sparse splitting, only a small number of nodes in the network are Multicast Capable, and the rest of nodes are Multicast Incapable (MI). An MI node can forward an input signal to only one output port. Thus, it cannot serve as a branching node of a light-tree. The sparse-splitting constraint certainly complicates the problem of efficient multicast routing in WDM networks.

Existing multicast routing solutions in sparse-splitting WDM networks can be classified into source-based trees [2-3], and MC-based trees [4-5], depending on how the light-trees are constructed. For source-based multicast schemes, lighttrees are built in a way similar to traditional IP multicast trees, with modifications to the routing algorithm for adapting to the sparse-splitting constraint. For MC-based schemes, a light-tree, called MC tree, connecting the MC nodes for a multicast group is first constructed. Each MI node for the group then separately joins the nearest MC node which is already on tree. MC-based schemes outperform source-based tree schemes in terms of better resource utilization, better support for dynamic membership, shorter tree setup latency, and improved scalability [5]. The differences in the existing mechanisms [2-5] are summarized as follows. There are four heuristics proposed in [2]. They generate multiple light-trees for a given multicast session, thus using multiple transmitters and multiple wavelengths for a session. In [3], the generated light-tree needs a single transmitter and a single wavelength per session. In [4], the authors proposed an all-optical multicast routing heuristic called AOMH, which generate a lighttree with a single transmitter and multiple wavelengths per session. It assumes that each MI node is equipped with a Tap-or-Continue (ToC) cross-connect. A ToC cross-connect cannot tap optical power from a wavelength channel during data forwarding through a router. The cross-connect that allows optical power to be tapped during data forwarding is called Tap-and-Continue (TaC). In [5], a virtual source-based multicast routing scheme is proposed. That scheme, however, needs to establish mutual connectivity among all MC nodes in advance.

The efficiency of an all-optical multicast routing mechanism can be evaluated with respect to the cost of the tree spent for the session. Such cost includes the wavelength channel cost and the number of different wavelengths used per session (i.e., the maximum number of different wavelengths per link used for a session). Here the wavelength channel cost is defined as the total number of wavelength channels used over all links 


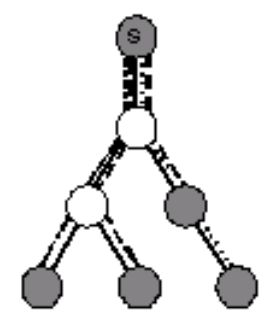

(a)

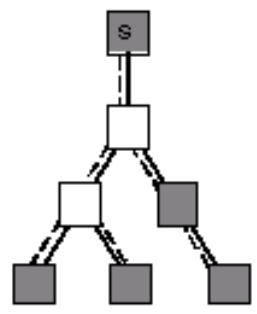

(b)

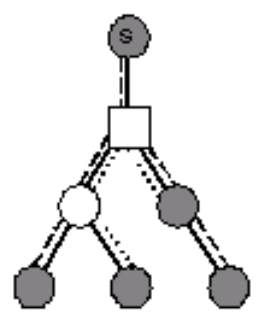

(c)

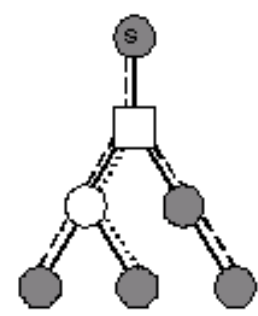

(d)

Fig. 1. Wavelength resources required by a light-tree under different network configurations. (a) All the nodes support ToC, (b) all the nodes support light-splitting, (c) sparse-splitting, with MI nodes supporting ToC, and (d) sparse-splitting, with MI nodes supporting TaC.

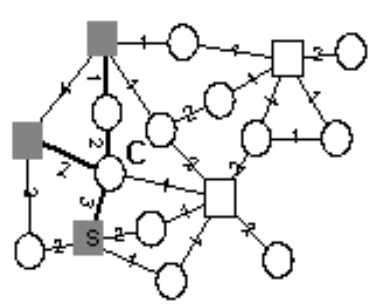

(a)

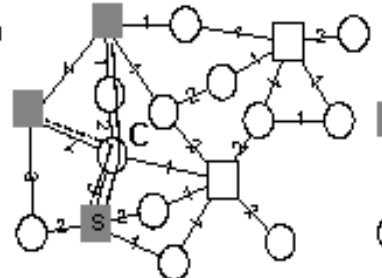

(b)

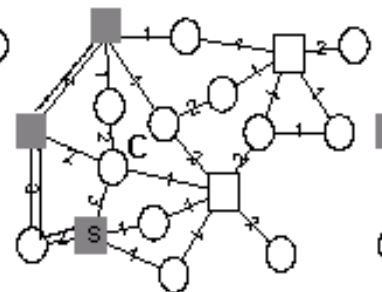

(c)

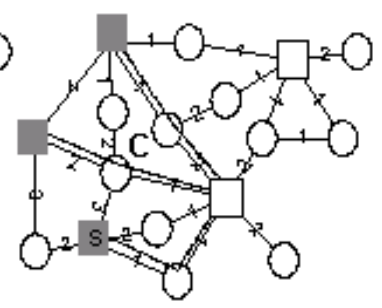

(d)

Fig. 2. Different rerouting approaches to constructing a multicast tree. (a) Network topology, (b) AOMH, (c) MemberOnly, and (d) optimal light-tree.

of the tree (i.e., the total number of branches of the multicast tree). Fig. 1 gives the cost of a multicast-tree under different network configurations, where a circle denotes an MI node and a square is an MC node; solid lines represent physical connections between nodes and dashed lines are light-paths for data transmission. Node $s$ is the source node and the other shaded nodes are the destinations. Fig. 1 (a) shows a multicast session using multiple independent light-paths in a network which consists only of ToC nodes. Thus, it needs four different wavelengths and eleven wavelength channels for the session. In Fig. 1(b), a light-tree, instead of multiple light-paths, in an all-MC network is constructed to support the multicast session. Thus, there is only one transmitter, one wavelength, and six wavelength channels. In Fig. 1(c), a light-tree is constructed in a sparse-splitting network with ToC MI nodes, and thus two different wavelengths and eight wavelength channels are required. In Fig. 1(d), a light-tree is constructed in a sparse-splitting network with TaC MI nodes. Thus, it uses two different wavelengths and seven wavelength channels. In Fig. 1, the cost for a multicast session can be greatly reduced if the nodes are multicast capable. Further cost reduction can be achieved if $\mathrm{MI}$ nodes support $\mathrm{TaC}$ instead of ToC. However, TaC operations may not be supported in most of today's wavelength switch architectures unless an add-drop multiplexer is used to receive packets through $\mathrm{O} / \mathrm{E}$ and forward copies through E/O [4]. Thus, for all-optical multicast, it is more reasonable to assume that MI nodes support ToC, rather than $\mathrm{TaC}$.

Most of the existing sparse-splitting multicast routing schemes take two steps for tree construction. First, an existing Steiner tree heuristic [6] is applied to the network so that an initial tree is obtained without taking the sparse-splitting constraint into account. Then, some rerouting procedure is adopted to refine the tree (see [2-4] for example). As a result, the cost of the resulting tree is as high as the cost of the original tree constructed by a Steiner tree heuristic plus some additional rerouting cost. Fig. 2 shows the multicast trees constructed by some existing work. Again, a circle represents an MI node, and a square denotes an MC node. Node $s$ (shaded) is the source node and the other shaded nodes are the destinations; routes are depicted as dashed lines. Fig. 2 (a) gives the initial network topology. The set of bold solid lines indicates the routing without considering the sparse-splitting constraint, and forms a minimum weight Steiner tree rooted at node $s$ with a cost of 8 . However, this is not a valid light-tree because the branching node $c$ is incapable of splitting signals, and additional rerouting is required. Fig. 2 (b) depicts a lighttree with a cost of 11 by AOMH [4], and Fig. 2 (c), a light-tree with a cost of 9 by MemberOnly [2] (i.e., the best heuristic in [2]). Fig. 2 (d) shows the minimum cost light-tree with a cost of 8 for the multicast session. This tree, however, cannot be obtained by any of the existing rerouting approaches. In summary, even though existing Steiner tree heuristics can guarantee a tight ratio bound (i.e., the performance metric ratio between the tree generated by the heuristic and the optimal tree), the additional rerouting cost imposed by them may degrade the performance. We will come back to this performance issue later in the simulation section.

In this paper, we propose a new multicast routing algorithm to construct MC-based light-trees without an additional rerouting procedure for all-optical networks. Considering the limited number of wavelength channels supported per fiber, we attempt to minimize the "wavelength channel" cost of a lighttree. For the MC-based multicast routing scheme, the cost of a light-tree is dominated by the cost of the corresponding $\mathrm{MC}$ tree, which accounts for the MC-tree cost plus the cost of 


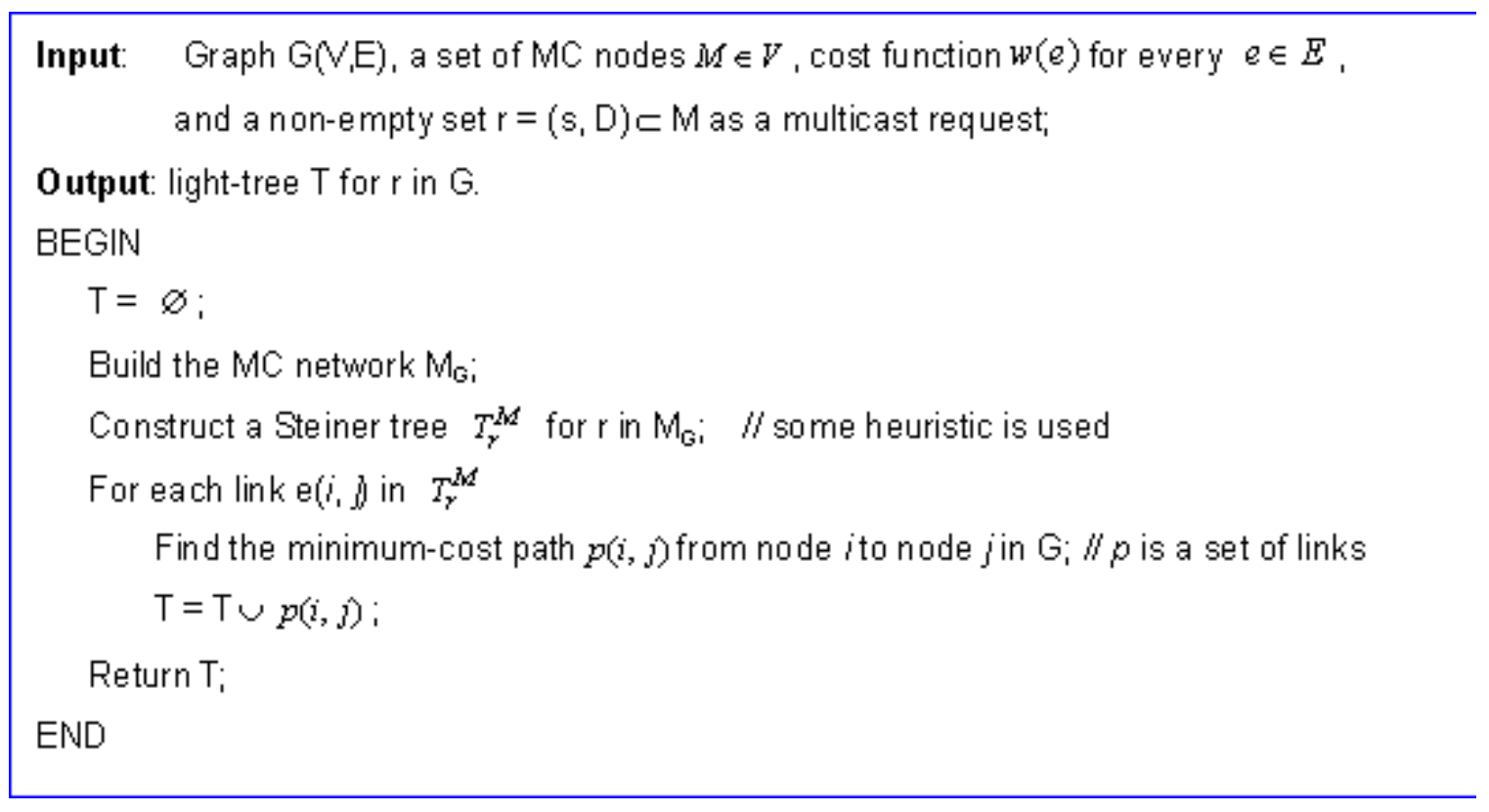

Fig. 3. The procedure of an MC-tree construction with ANT.

each light-path connecting an MI node to the MC tree. The design goal of the proposed method is twofold: i) to choose a proper set of MC nodes as on-tree nodes. The choice not only affects the cost of the MC tree, but also affects the cost of the light-paths connecting the MI nodes to the MC tree. ii) to determine the minimum-cost light-tree for a given set of on-tree MC nodes. For i), we design two MC-node selection policies; for ii), we propose a new mechanism that builds the minimum-cost light-tree without rerouting. We also briefly describe how to extend our mechanism to support dynamic group membership such that each member can freely join or leave a session without affecting the other members.

The rest of the paper is organized as follows. In Section II, we design a mechanism to construct a minimum-cost MC tree, and propose two efficient schemes to select on-tree MC nodes. In Section III, the performance of the proposed mechanism is evaluated via simulations. Finally, the paper is concluded in Section IV.

\section{Construction of Light Trees}

In this section, we will describe the proposed mechanism for all-optical multicast routing in sparse splitting WDM networks. We adopt an MC-based scheme for tree construction, but eliminate extra rerouting procedures adopted by most of the existing work. In what follows, we first describe the procedure that constructs an MC tree with minimum cost for a given multicast session, and then propose two different schemes to select MC nodes for MI nodes to join the tree. Finally, we extend our mechanism to support dynamic multicast.

The optical network we consider is assumed with two types of cross-connects: MI and MC nodes. MI nodes perform ToC operations during data forwarding; MC nodes support light splitting to forward data to multiple outgoing wavelength channels. MC nodes are assumed to be equipped with wavelength converters. Multicast receivers may be connected to the light-tree via MC nodes or MI nodes.

\section{A. Minimum-Cost MC-Tree}

For MC-based multicast routing schemes, the resulting light-tree $T$ consists of an MC tree $T_{M C}$ and a set of lightpaths $L_{T}$ connecting the remaining destinations via MI nodes to the MC tree. The wavelength channel cost of tree $T$, denoted by $\mathrm{WC}(T)$, includes the wavelength channel cost of the $\mathrm{MC}$ tree plus the cost of the light-paths, i.e., $W C(T)=$ $W C\left(T_{M C}\right)+\sum_{L_{T}^{i} \in L_{T}} W C\left(L_{T}^{i}\right)$. Our goal is to develop an efficient algorithm which minimizes $W C\left(T_{M C}\right)$. This problem is, however, NP-hard because it can be reduced from the standard Steiner tree problem even when the sparse-splitting constraint is relaxed.

The typical solution to light-tree construction in existing work [2-5] is to combine an existing IP multicast routing algorithm with a rerouting procedure. Our mechanism does not incur extra rerouting for light-tree construction. An MC network $\mathbf{M}_{\mathbf{G}}$ is derived from the original network $\mathbf{G}$ as follows.

1) All the $\mathrm{MC}$ nodes in network $\mathbf{G}$ are included as its vertex set, say W.

2) If there is a path between two $\mathrm{MC}$ nodes in $\mathbf{G}$, these two $\mathrm{MC}$ nodes are connected in $\mathbf{M}_{\mathbf{G}}$.

3) The link cost from node $i$ to node $j$ in $\mathbf{M}_{\mathbf{G}}$ is set to the cost of the minimum-weight path from node $i$ to node $j$ in $\mathbf{G}$, for all $i, j \in W$.

In Appendix A, we prove that finding an MC tree whose wavelength channel cost is minimum is equivalent to finding a Steiner tree in the corresponding MC network. In other words, once an auxiliary MC network is constructed, we can obtain the minimum-cost $\mathrm{MC}$ tree for a multicast session by applying any existing Steiner tree heuristic to the MC network. Our solution is summarized as follows. It first builds an auxiliary MC network $\mathbf{M}_{\mathbf{G}}$ from the original network $\mathbf{G}$, and then applies an existing Steiner tree heuristic to $\mathbf{M}_{\mathbf{G}}$ to obtain a minimum-cost $\mathrm{MC}$ tree. The minimum-cost $\mathrm{MC}$ tree is 


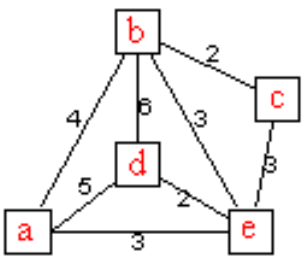

(a)

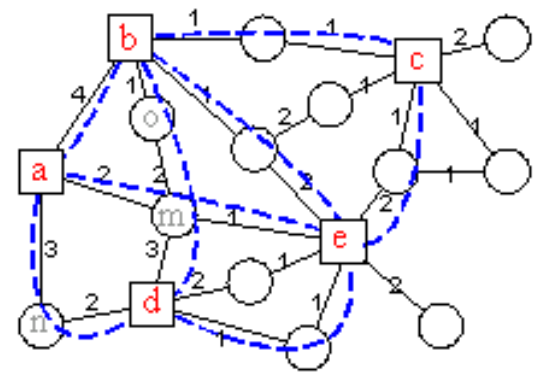

(b)

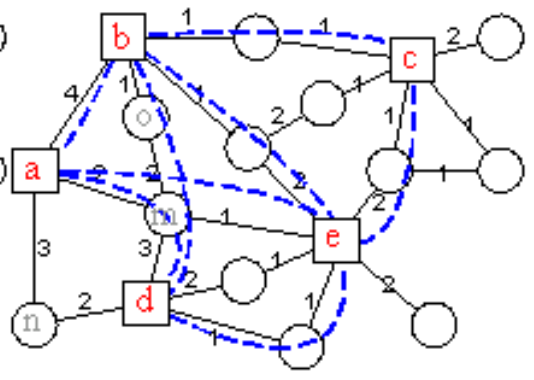

(c)

Fig. 4. A mapping between an edge in an MC network and a shortest path in the original network. (a) The MC network $\mathrm{M}_{\mathrm{G}}$, (b) one possible set of light path (dotted lines), (c) another possible set of light-paths (the dotted lines).

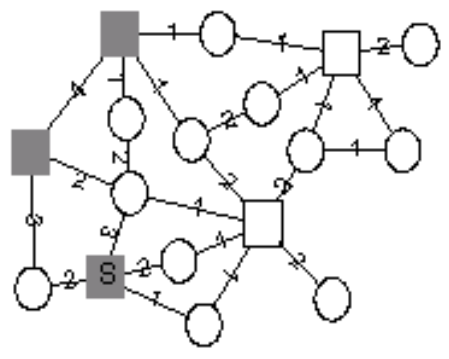

(a)

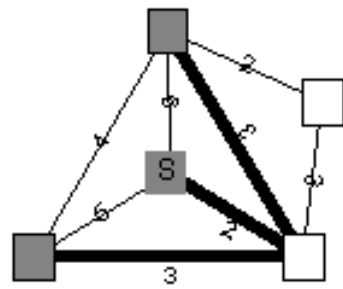

(b)

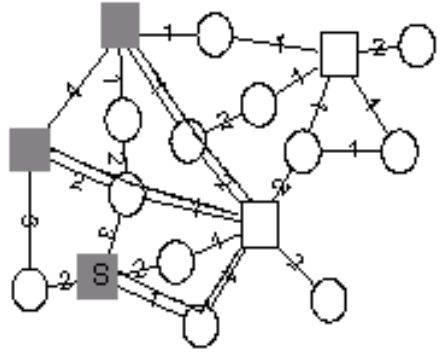

(c)

Fig. 5. An example of ANT operation. (a) Network topology, (b) the corresponding MC network and resulting Steiner tree, and (c) resulting light-tree.

then transformed back to the original network to yield the resulting light-tree. We call this approach "Auxiliary Network Transformation (ANT)," which is summarized as follows.

1) The $M C$ network $\mathbf{M}_{\mathbf{G}}$ of the original network $\mathbf{G}$ is determined.

2) A Steiner tree heuristic is applied to $\mathbf{M}_{\mathbf{G}}$ to generate a minimum-cost tree $\mathbf{T}_{\mathbf{R}}$ for multicast session $\mathbf{R}$.

3) The resulting light-tree $\mathbf{T}$ is obtained by substituting each link, say $(i, j)$, in $\mathbf{T}_{\mathbf{R}}$ with the corresponding minimum-cost path from node $i$ to node $j$ in $\mathbf{G}$.

Each edge $(i, j)$ in $\mathbf{M}_{\mathbf{G}}$ is mapped to a shortest path from node $i$ to node $j$ in the original network $\mathbf{G}$, as shown in Fig. 4. We note that i) the shortest path from $i$ to $j$ may not be unique (for example, Figs. 4(b) and 4(c) give two alternatives), and ii) two edges in $\mathbf{M}_{\mathbf{G}}$ may contend for the same wavelength link in $\mathbf{G}$ (for example, path $<\mathbf{a}, \mathbf{m}, \mathbf{d}>$ contends for the same link with paths $\langle\mathbf{a}, \mathbf{m}, \mathbf{e}\rangle$ and $\langle\mathbf{b}, \mathbf{o}, \mathbf{m}, \mathbf{d}\rangle$ in Fig. 4(c)). The contention problem with ii) may be inevitable due to the nature of sparse-splitting networks. Although it will not affect the wavelength channel cost, it increases the maximum number of wavelengths used per link. To alleviate this problem, we propose a simple policy to select among multiple shortest-path routes when i) occurs:

1) Reserve the light-path with the least possible shortestpath routes.

2) When there are multiple shortest-path routes available, reserve the wavelength link suffering the least contention.

With this remedy, we may reduce the number of contention wavelength links when multiple shortest-path routes are available in the network. Take Fig. 4 for example. All edges in
$\mathbf{M}_{\mathbf{G}}$ are mapped to one possible shortest path in $\mathbf{G}$ except $\langle\mathbf{a}, \mathbf{d}\rangle$, where both $\langle\mathbf{a}, \mathbf{m}, \mathbf{d}\rangle$ and $\langle\mathbf{a}, \mathbf{n}, \mathbf{d}\rangle$ in $\mathbf{G}$ are shortest-path routes for $\langle\mathbf{a}, \mathbf{d}\rangle$. Therefore, the light-path from $a$ to $d$ is reserved last, thus avoiding contentions on links $\langle\mathbf{a}, \mathbf{m}\rangle$ and $\langle\mathbf{m}, \mathbf{d}\rangle$ due to selecting $\langle\mathbf{a}, \mathbf{n}, \mathbf{d}\rangle$.

Fig. 3 summaries the algorithm ANT, and Fig. 5 demonstrates how ANT works. Again, node $s$ is the source node and the other shaded nodes are the destinations. The corresponding MC network is shown in Fig. 5(b), and the resulting MC tree is shown in Fig. 5(c). Note that the effectiveness of the Steiner tree heuristic used significantly affects the cost of the resulting tree. Thus, the cost of MC tree generated by ANT is bounded by the error-ratio of the Steiner tree heuristic in use.

\section{B. Two MC Node Selection Policies}

We have described how to determine a minimum-cost MC tree using ANT for a multicast session, provided that the set of MC nodes to be included is given. We now turn to the issue of selecting MC nodes to be included to a tree. As described in Section I, the number of MC nodes included in an MC tree not only affects the cost of the MC tree itself, but also the cost of the MI nodes connected to the MC tree. The more the MC nodes are involved in an MC tree, the more the resource is consumed. On the other hand, the light-path cost from an MI node to the MC tree will be be reduced if the MC tree spans the network. Therefore, the policy of selecting MC nodes to be included to the tree is the key to determining the cost of the resulting light-tree. It is hard to optimally select a set of $\mathrm{MC}$ nodes (to construct an MC tree) which minimizes the cost of the resulting light-tree for a given multicast session. Since this problem is equivalent to finding an MC tree with 


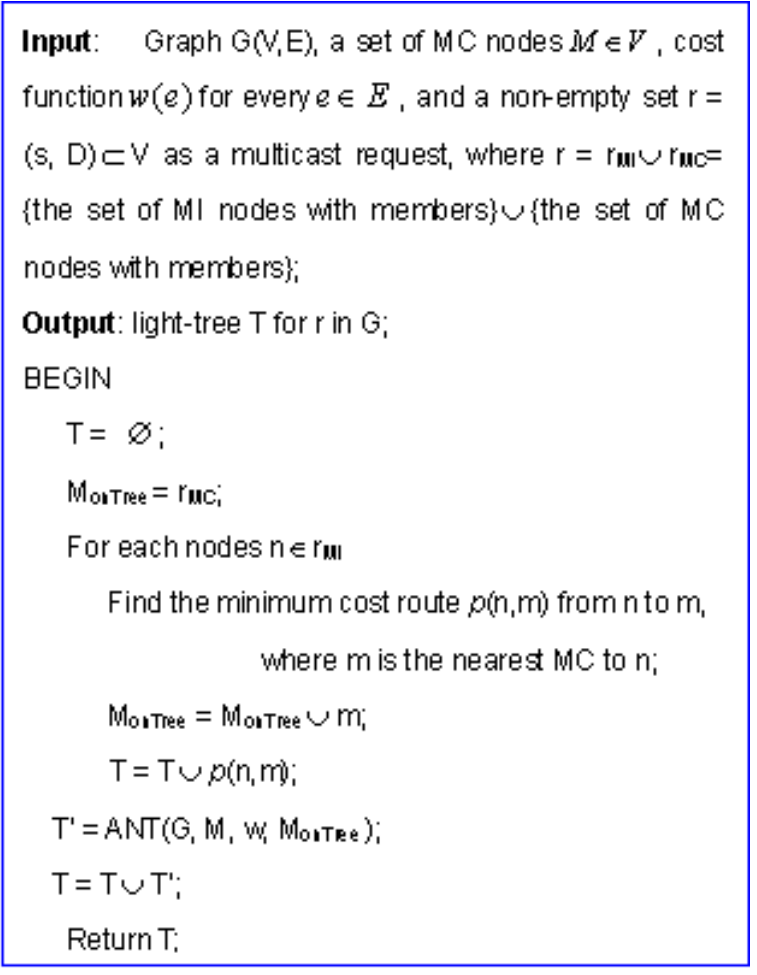

(a) OTMCF
Input: Graph $G(V, E)$, a set of MC nodes $M \in V$, cost function $w(e)$ for every $e \in E$, a non-empty set $r=$ $(s, D) \subset V$ as a multicast request, where $r=r_{m \mid} \cup r_{m}=$ the set of MI nodes with members\} $\cup$ the set of MC nodes with members\}; Output: light-tree $\mathrm{T}$ for $\mathrm{r}$ in $\mathrm{G}$; BEGIN

$$
\begin{aligned}
& \text { Mortree }=r u c ; \\
& T=A N T\left(G, M, W_{1} \text { Mor tree }\right) ; \\
& M_{\text {outree }}=M C_{T i} \quad \text { I/ MC nodes on T }
\end{aligned}
$$

For each nodes $n \in r_{m I}$

Find the minimum cost route $p$ from $n$ to Mor Tree; $\mathrm{T}=\mathrm{T} \cup p_{i}$

Return T; END

Fig. 6. Two MC node selection policies.

minimum wavelength channel cost if all the group members are connected to MC nodes, again, it is an NP-hard problem. Thus, it is required to find an efficient approach that chooses a proper subset of MC nodes in the network to be on-tree MC nodes.

Next, we propose two efficient approaches to selecting MC nodes as on-tree MC nodes, based on two different design philosophies.

1) On-Tree MC node First (OTMCF): this approach attempts to minimize the cost of an MC tree. An MC tree is constructed by first including all the MC nodes to which group members are directly connected, and then expanded as follows. The MI nodes to which the remaining members are directly connected join the tree through the nearest on-tree MC nodes.

2) Nearest MC node First (NMCF): this approach attempts to minimize the costs of MI nodes which join the MC tree. The set of MC nodes used to construct an MC tree consists of all the MC nodes directly connecting to the destinations of the group and the MC nodes which are the nearest to the MI nodes connecting to the remaining destinations. NMCF expands on-tree MC nodes in such a way that each MC node nearest to each MI node in the group must also be on-tree.

Fig. 6 summarizes the operations of light-tree construction for OTMCF and NMCF, together with ANT described in Section II-A. In Section II-C, we will explore the possibility of light-tree construction without going through the complexity of graph transformation as in ANT.

\section{Reduced Complexity of an MC-Tree}

Recall from Section II-A that an auxiliary MC network is used to construct light-trees. This approach, however, requires a time complexity of $\mathrm{MN}^{2}$ for tree construction, where $M$ is the number of MC nodes in the network, and $N$ is the total number of nodes in the network. This complexity stems mainly from the graph transformation between the original network and the auxiliary MC network. Taking a closer look at this approach, we find that the wavelength channel cost between any two MC nodes on the MC-tree in the MC network equals the cost of the minimum-weight path between them on the resulting light-tree in the original network. This implies that if a Steiner tree heuristic such as Shortest Path Heuristic (SPH) [7] is applied to the tree construction, under which the path between any two nodes on the determined tree is always in the minimum-cost path between them, the same results can be obtained without explicitly transforming to the MC network as in ANT. SPH works as follows. The multicast tree is first initialized with an arbitrary multicast member. Then, the tree grows by successively adding the next nearest multicast member to the tree through the shortest path from the member to the tree. The algorithm terminates when all the multicast members have joined the tree.

Figs. 7 and 8 demonstrate how the same light-tree is produced with these two approaches for a given multicast session in a network, using SPH as the Steiner tree heuristic in the example. Fig. 7 depicts how to construct a light-tree with ANT plus SPH. The corresponding MC network is first constructed. Initially the tree contains the source node only, 


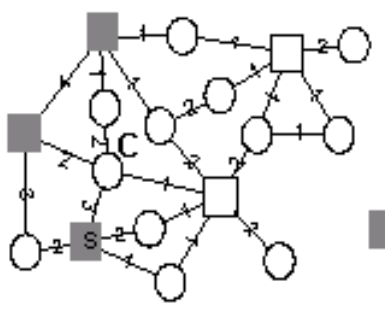

(a)

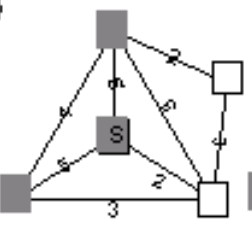

(b)

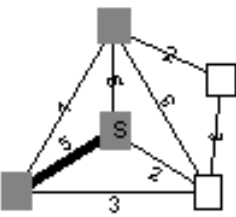

(c)

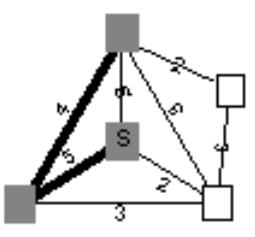

(d)

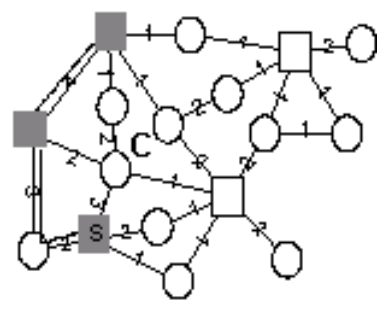

(e)

Fig. 7. Light-tree constructed with ANT, using SPH as the Steiner tree heuristic.

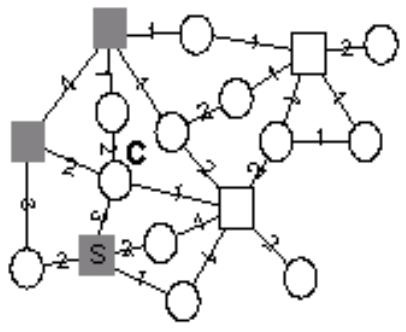

(a)

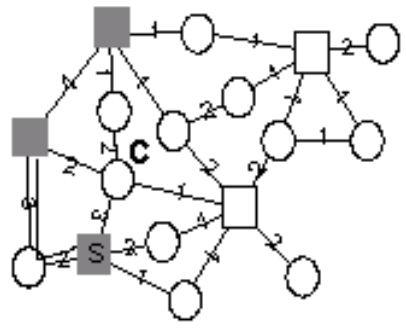

(b)

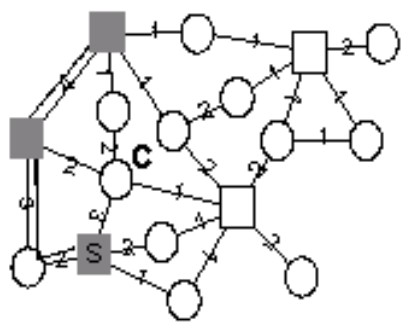

(c)

Fig. 8. Light-tree constructed by directly applying SPH to the original network.

as in Fig. 7(b). Then the tree grows sequentially as described above until all the multicast members are on-tree, as shown in Figs. 7(c) and (d). Finally the resulting tree is transformed back to the original network to yield the light-tree, as in Fig. 7(e). SPH, as illustrated in Fig. 8, need no auxiliary MC network to obtain the same tree. The resulting light-tree in Fig. 8(c) is exactly the same as that in Fig. 7(e). At each step, SPH always finds the nearest multicast member through the shortest path to the tree, thus eliminating the use of the MC network.

From the examples above, SPH can generate the same lowcost MC tree as ANT but without using an auxiliary MC network. Note that SPH is a special case of Steiner tree algorithms, thanks to its particular characteristic of connecting members to the tree via shortest paths. Most existing Steiner tree heuristics, such as KMB [8], ADH [9], and many QoS multicast algorithms [10], cannot generate an MC tree without rerouting. Thus, the efficiencies and characteristics of these algorithms may not be preserved after rerouting. With ANT, each of these algorithms can be used to generate the minimumcost MC tree without any performance degradation.

\section{From Static to Dynamic Multicast}

Finally, our mechanism is extended to efficiently support dynamic multicast for the proposed MC-based light-trees. Dynamic membership can be implemented in the all-optical domain based on existing architectures of Multicast-Capable optical cross-connects such as VWP-OXC [11]. Both OTMCF and NMCF can be extended in a straightforward way to support dynamic group membership. The only obstacle is that the proposed MC tree construction algorithm described so far has been assumed static. A simple solution is to adopt some tree rerouting technique to rebuild the tree when members are allowed to freely join or leave the tree without affecting the other members. This solution, however, is impractical as it would incur too much overhead during tree maintenance. Here we propose another solution. Instead of modifying the proposed static tree construction, we employ an on-line Steiner tree heuristic such as GREEDY[12] together with the proposed MC node selection policy to dynamically build the lighttree. GREEDY is designed to perturb the existing tree as little as possible. For each "add" request, the new member is connected to the nearest tree node through the shortest path. For each "delete" request, only the leaf nodes are deleted. If this deletion creates a nonmember leaf, the new leaf is also deleted. This process repeats until no nonmember-leaf nodes remain in the network. Fig. 9 illustrates the operations when GREEDY and NMCF are combined to build the lighttree dynamically. Initially, the multicast group includes source node $S$ only, as shown in Fig. 9(a). Node $A$ joins the group by first connecting to the nearest MC node, which is $m$. Node $m$ is then connected to the nearest on-tree MC node, which is $S$. Nodes $B$ and $C$ then join this group subsequently as shown in Figs. 9(c) and 9(d), respectively. In Fig. 9(e), node $A$ leaves the group and tears down the channels between $A$ and $m$. Node $m$ remain on-tree because node $C$ is still connected to the light-tree through node $m$.

Obviously, the resulting tree cost increases after a series of dynamic join/leave operations, as compared with that of static membership. Taking a closer look at dynamic NMCF, we find that the cost of MI nodes which join the MC tree is equal to that in static NMCF. NMCF guarantees that each MI node joins the MC-tree via the nearest MC node in the network. The only difference between static and dynamic NMCF is in the cost of the MC tree. With ANT proposed in Section II-A, the $\mathrm{MC}$ tree problem can be treated as a Steiner tree problem. More sophisticated dynamic algorithms for the Steiner tree, such as WEIGHTED GREEDY [12], may be applied via ANT. 


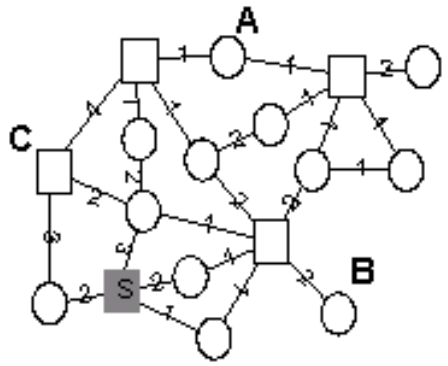

(a)

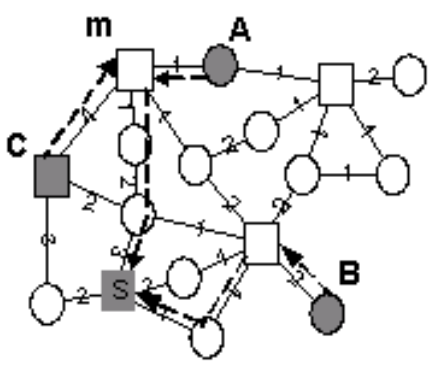

(d)

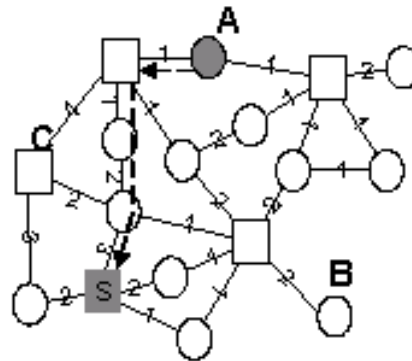

(b)

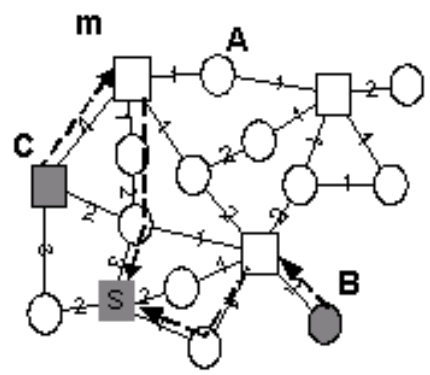

(e)

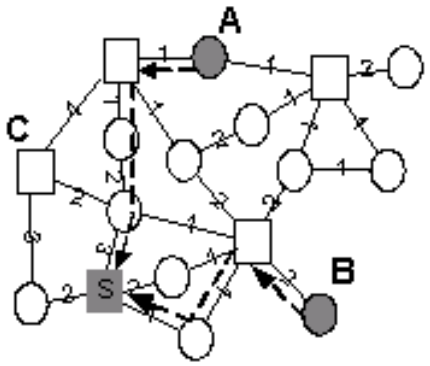

(c)

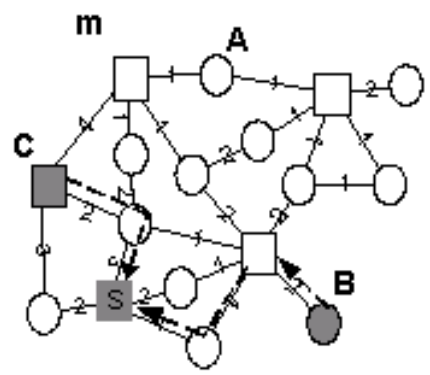

(f)

Fig. 9. An example of dynamic NMCF: (a) initially the group includes only S, (b) A joins the group, (c) B joins the group, (d) C joins the group, (e) A leaves the group, and (f) the minimal cost light-tree with static membership.

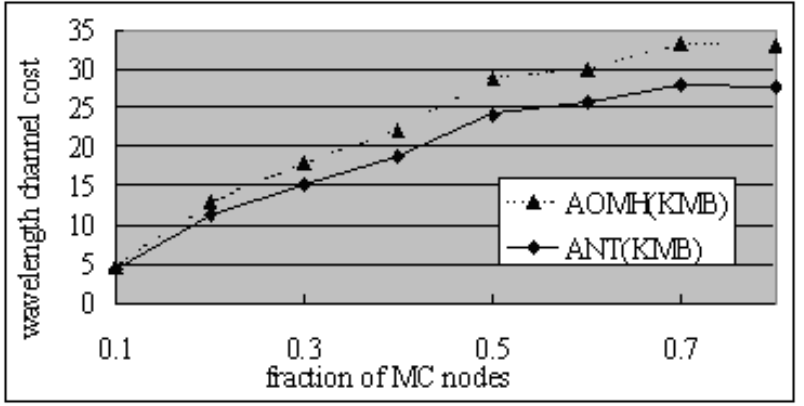

Fig. 10. Wavelength channel cost of an $\mathrm{MC}$ tree.

However, they are not applicable to OTMCF. With OTMCF, the set of on-tree MC nodes to which each MI node attempts to connect may vary over time due to dynamic membership.

\section{Performance Evaluation}

In this section, we evaluate the performance of our proposed mechanism and existing work AOMH and MemberOnly via simulations. Our mechanism is an MC-based approach, that is, building an $\mathrm{MC}$ tree first and expanding to a light-tree to reach all the members. To show the benefit of using ANT to construct an $\mathrm{MC}$ tree, we first compare the costs of our constructed MC trees and AOMH's (previous work on the MC-based approach). Then the whole light-tree costs of our mechanisms are evaluated and compared with AOMH's and MemberOnly's (previous work on the source-based approach). Note that for MC-based approaches, we only consider AOMH [4] because other MC-based mechanisms are all based on different network configurations.

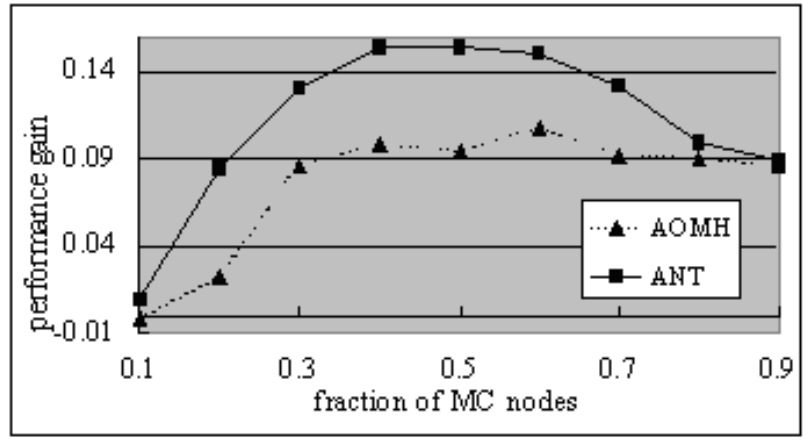

Fig. 11. Performance gain of KMB over DDMC.

In this simulation, we generate a flat network consisting of 50 nodes with GT-ITM [13]. Each MC node in the network is equipped with both capabilities of splitting and wavelength conversion. The cost of each wavelength channel on each link is assigned a value of one. We use KMB[8] and DDMC[14] as the Steiner tree heuristics for both ANT and AOMH in the simulations. KBM is a 2-approximate Steiner tree heuristic with a complexity of $\mathrm{O}\left(|\mathrm{M}||\mathrm{V}|^{2}\right)$, where $\mathrm{O}(|\mathrm{M}|)$ is the size of the group and $\mathrm{O}(|\mathrm{V}|)$ is the total number of nodes in the network. The complexity of DDMC is $\mathrm{O}(|\mathrm{E}| \log |\mathrm{V}|)$, where $\mathrm{O}(|\mathrm{E}|)$ is the number of links in the network. Thus, its complexity is lower than KMB's, but its worst-case error ratio cannot be guaranteed.

\section{A. Wavelength Channel Cost}

1) MC-tree construction: First, we compare ANT with AOMH using the same number of MC nodes. With AOMH, an MC tree is built by first applying some Steiner tree 


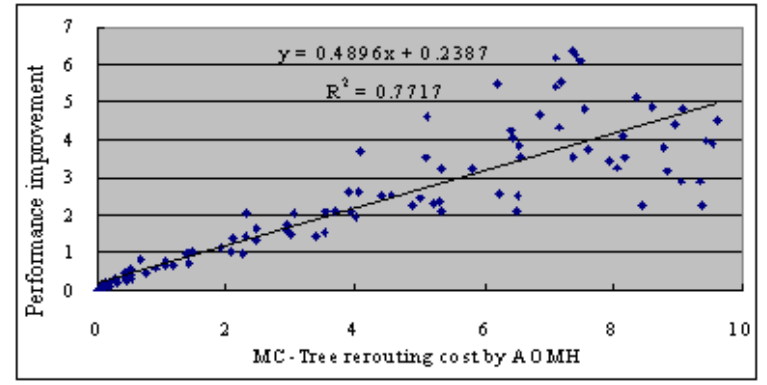

(a) $\mathrm{KMB}$

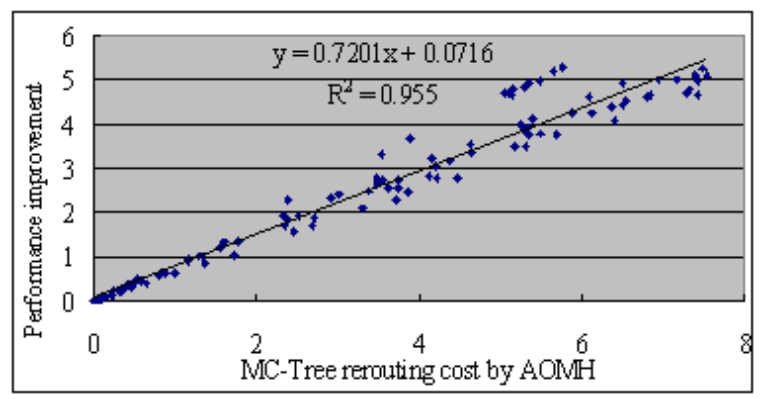

(b) DDMC

Fig. 12. Performance improvement of ANT over AOMH.

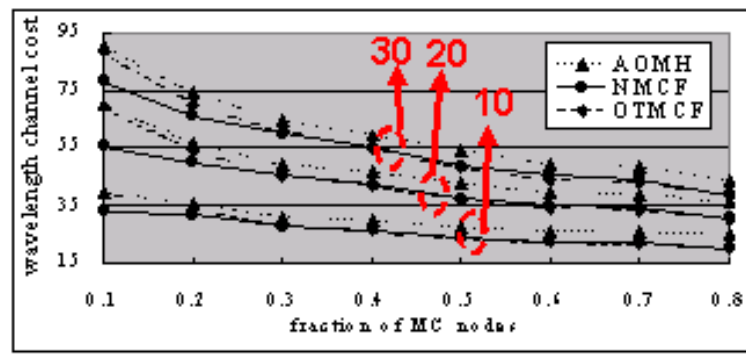

(a) DDMC

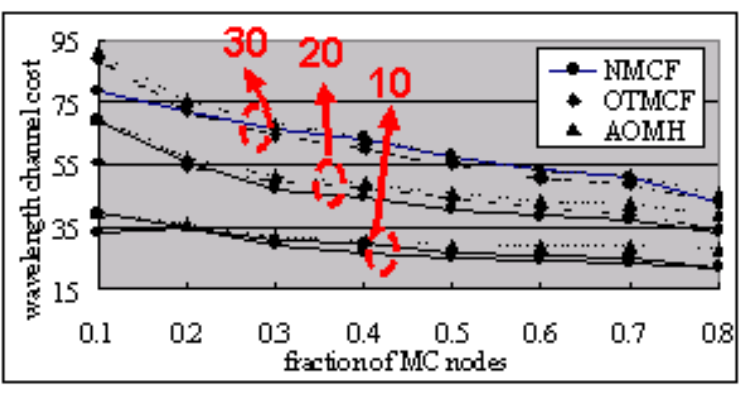

(b) KMB

Fig. 13. Wavelength channel cost of a light-tree as a function of the fraction of MC nodes.

heuristic and then rerouting to be a valid light-tree [4]. In this simulation, KMB is the Steiner tree heuristic for both of ANT and AOMH. We randomly generate 200 multicast sessions with group size 20 in a 50-node network, and let ANT and AOMH find their trees for MC nodes in the group. Fig. 10 shows the average costs of the MC trees used for a multicast session for both mechanisms. We can see that ANT spends the least wavelength channel cost in all cases. For both approaches, the costs increase as the fraction of MC nodes increases. This is because more MC nodes may be included onto the MC tree as the fraction of MC nodes in the network grows. In fact, as the fraction of MC nodes increases, the cost of the resulting light-tree decreases, as shown in Fig. 13. We observe that about $17 \%$ cost reduction can be achieved with ANT as compared with AOMH when the fraction of MC nodes is high.

Next, we study the impact of Steiner tree heuristics on the performance of these two protocols. We first adopt KMB for both approaches, and then switch to DDMC to observe the change in tree costs. Fig. 11 shows that the average performance gain obtained by KMB is over DDMC. The performance improvement of ANT is sharper than AOMH when a better heuristic (i.e., $\mathrm{KMB}$ ) is used, especially when the fraction of MC nodes is not so high. This is because AOMH's rerouting procedure may degrade the performance of the Steiner tree heuristic, while there is no such problem with ANT thanks to without a rerouting procedure.

We further investigate the impact of rerouting on the performance of ANT and AOMH, using two Steiner tree heuristics. Fig. 12 plots the performance gains of ANT and AOMH, with different rerouting cost incurred by AOMH. Each data point is generated under a different setting of fraction of MC nodes and group sizes. The performance gain obtained by ANT is over AOMH, and is almost proportional to the rerouting cost of AOMH. To study the linear relation between these two measurements, we calculate the regression line $L$, which has a minimal total square error between $L$ and every data point for both heuristics. For each regression line, the R-squared value (ranging from 0 to 1 ), which indicates the significance, is also calculated. The results show that ANT can reduce the rerouting cost incurred by AOMH by about $50 \%$ if DDMC is used. With KBM, the rerouting cost can be reduced by over $70 \%$. Therefore, the performance gain obtained by ANT thanks to no rerouting is more significant when KMB is used.

\section{B. Light-Tree Construction}

We next evaluate the performance of light-trees constructed by OTMCF, NMCF and $\mathrm{AOMH}$, varying the fraction of $\mathrm{MC}$ nodes and group sizes. Fig. 13 shows the average wavelength channel costs of light-trees for the three approaches with different Steiner tree heuristics. We show three sets of results, corresponding to group sizes of 10,20 , and 30 . In all cases, both OTMCF and NMCF have better performance than AOMH. NMCF outperforms OTMCF when the fraction of MC nodes is low, but OTMCF performs better when the fraction of MC nodes increases. This is because the cost of a light-tree is dominated by the cost of the MC tree when the fraction of $\mathrm{MC}$ nodes is high, and is dominated by the cost of MI nodes connected to the MC tree when the fraction of MC nodes is low.

With DDMC as the Steiner tree heuristic, NMCF outperforms OTMCF if the percentage of MC nodes is less than 


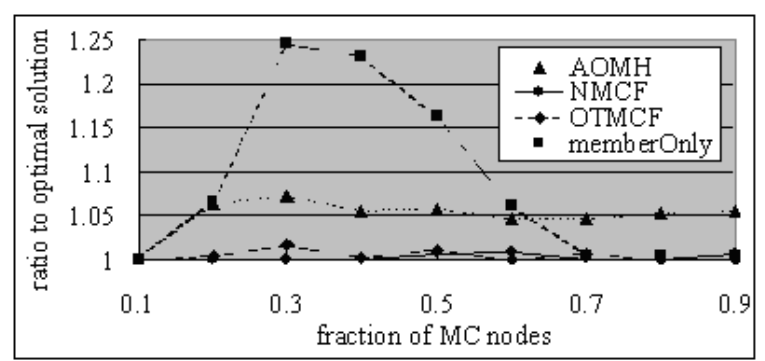

(a) Group size $=7$

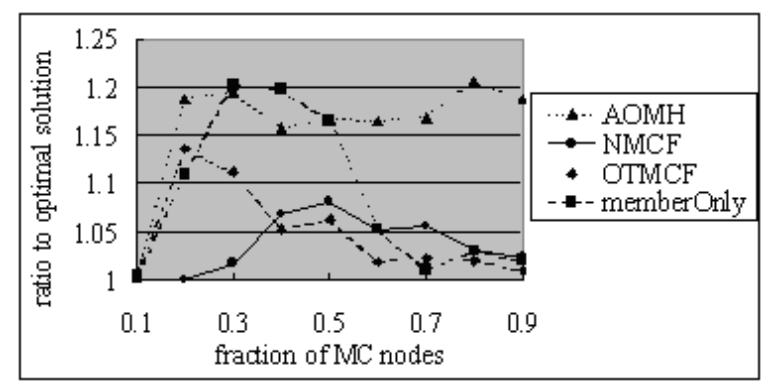

(b) Group size $=13$

Fig. 14. Wavelength channel cost compared to the optimal for different approaches.

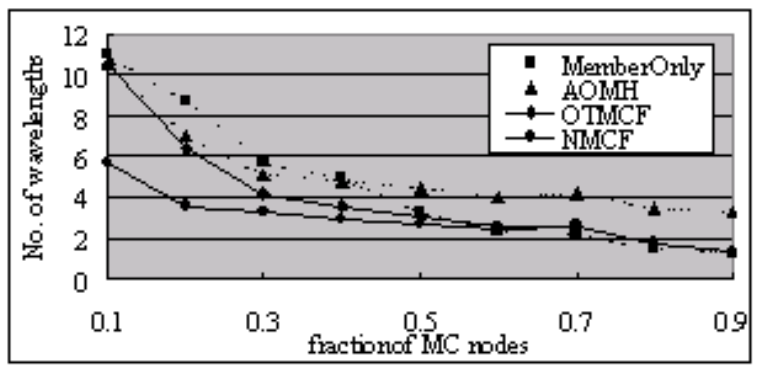

(a) Number of wavelengths

Fig. 15. Maximum number of wavelengths used per link with group size 5 .

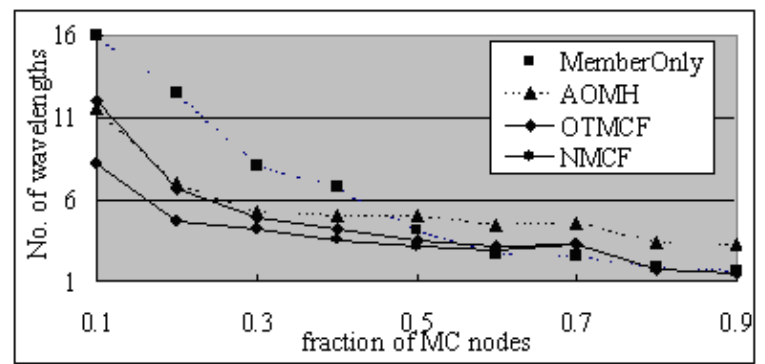

(a) Number of wavelengths

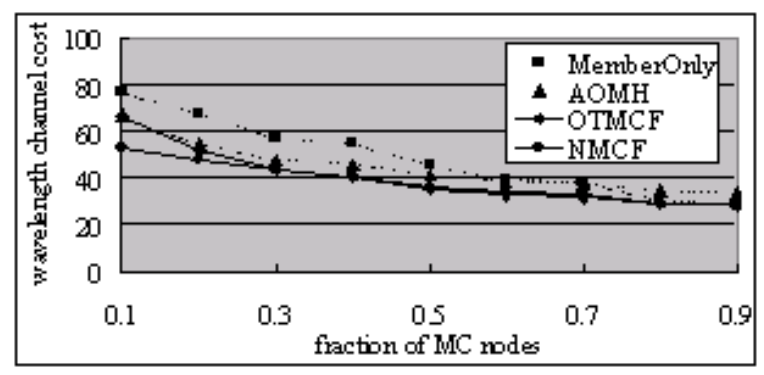

(b) Wavelength channel cost

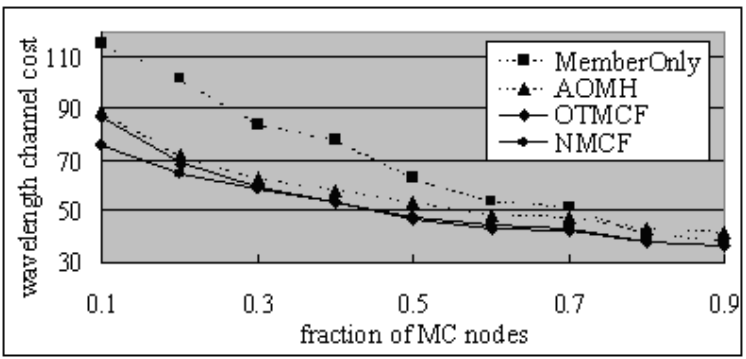

(b) Wavelength channel cost

Fig. 16. Maximum number of wavelengths used per link with group size 20 .

$20 \%$. With KMB, NMCF outperforms OTMCF by a great margin if the percentage of MC nodes is less than $30 \%$, and the performance advantage wanes as the percentage of MC nodes exceeds $30 \%$. Therefore, NMCF with KMB is a better choice to construct a light-tree in a sparse-splitting WDM network.

To further investigate the effectiveness of light-trees constructed by each approach, especially for observing the lighttree costs of our mechanisms compared with the optimal, we design an optimal algorithm using the brute-force mechanism to calculate the minimum cost of a light-tree. Due to the high computational complexity of the brute-force solution, we use a smaller network in this simulation. We randomly generate 200 multicast sessions with group sizes 7 and 13 in a 15 -node network. We then apply OTMCF, NMCF, AOMH, memberOnly, and the brute-force mechanism to construct light-trees and calculate the ratio of the tree cost of each approach to that of the brute-force optimal one. Fig. 14 shows the cost of each solution as a fraction of the optimal solution. Fig. 14 (a) shows the result with group size 7, and Fig.
14 (b), group size 13. It shows that the light-tree cost with NMCF is very close to the optimal solution (within $1 \%$ error ratio), especially when the group size is large (see Fig. 14 (b)). AOMH has a larger error ratio when the group size is small, but stays close to the optimal when the group size increases. With a small fraction of MC nodes, the sourcebased mechanism (i.e., MemberOnly) has a large error ratio, especially when the group size is large (see Fig. 14 (b)).

\section{Maximum Number of Different Wavelengths Used per Link}

We then compare the maximum number of different wavelengths used per link in a session for each approach. Again, we consider MemberOnly, AOMH, OTMCF, and NMCF, using $\mathrm{KMB}$ as the Steiner tree heuristic. Note that since MemberOnly is a source-based scheme, we modify it as follows. Instead of using the source node as the tree root, the MC node which is the nearest to the source in the group is selected as the root to meet our single transmitter assumption. The rest of the operation remains intact. 


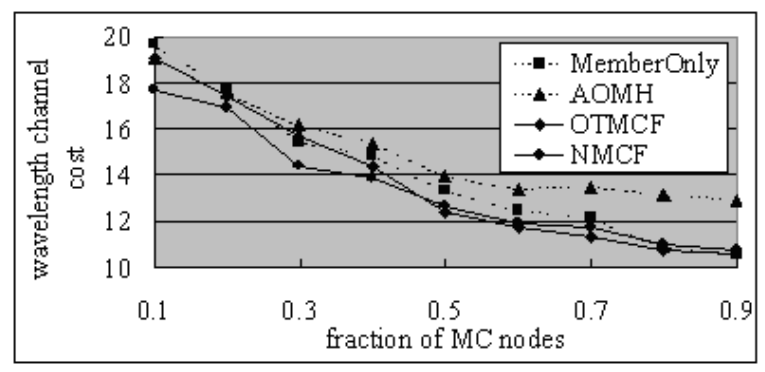

(a) Number of wavelengths

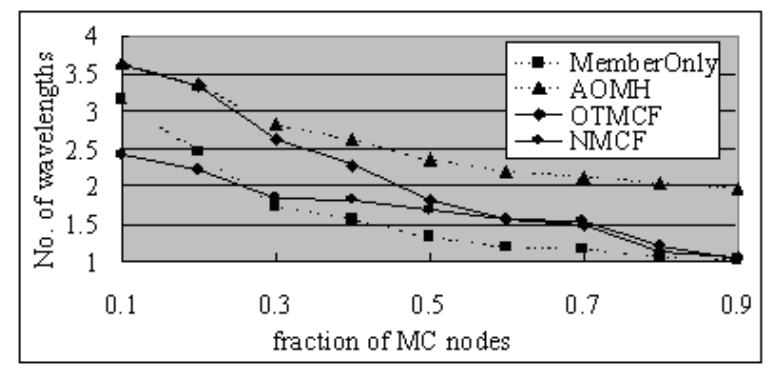

(b) Wavelength channel cost

Fig. 17. WMaximum number of wavelengths used per link with group size 30.

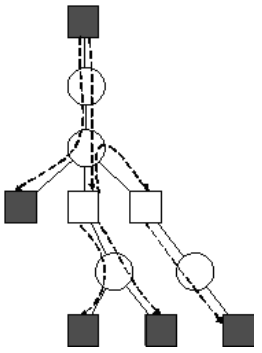

Fig. 18. A light-tree can be viewed as a set of light-paths connecting MC nodes.

Figs. 15-17 (a) show the maximum number of wavelengths used per link for the four approaches, with different group sizes. The wavelength channel cost of the light-tree for each approach is given in Figs. 15-17 (b) for comparison. It can be observed that the number of wavelengths required for a given light-tree always decreases as the fraction of MC nodes increases for all approaches. When the percentage of MC nodes in the network exceeds 50\%, MemberOnly can construct light-trees efficiently in terms of the number of wavelengths required. However its performance degrades rapidly when the percentage of MC nodes in the network becomes small, especially with larger group sizes. In most cases, its required number of wavelengths is more than twice that required by NMCF. NMCF always outperforms MemberOnly except for some cases with group size 5 . As for the wavelength channel cost, NMCF always uses the least number of wavelength channels. MemberOnly builds light-trees with a large number of wavelength channels, especially when the group size is large. Thus we can conclude that NMCF is the most efficient way of constructing light-trees under various situations.

\section{CONCLUSION}

In this paper, we propose two mechanisms to construct MC-based light-trees with minimum wavelength channel cost for multicast sessions in sparse-splitting optical networks. We start with static group membership and then extend to support dynamic multicast. The traditional solution to this problem is to combine a Steiner tree heuristic with some rerouting mechanism to refine the tree. Our mechanism eliminates the requirement of rerouting. We prove that the problem of minimizing the channel cost of an MC tree is equivalent to the standard Steiner tree problem. We then design an approximation algorithm to construct a minimum-cost $\mathrm{MC}$ tree. We propose two light-tree construction algorithms based on two different on-tree MC nodes selection philosophies. Finally, we compare the performance of our mechanism with AOMH and MemberOnly via simulations. The results show that NMCF with $\mathrm{KMB}$ is the best choice for multicast in sparse splitting optical networks. In all cases, our mechanism produces the least wavelength channel cost and uses the smallest number of wavelength channels per link, as compared with existing work.

\section{APPENDIX I \\ Minimum Wavelength-Channel-Cost MC Tree PROBLEM}

In this appendix, we formulate the minimum wavelengthchannel-cost $\mathrm{MC}$ tree problem and show its equivalence to the standard Steiner tree problem.

\section{A. Notations}

1) Let $\mathrm{WC}(\mathrm{T})$ denote the wavelength channel cost of a light-tree $\mathbf{T}$, and $W C_{T}(p, q)$, the wavelength channel cost of connecting nodes $p$ and $q$ on $\mathbf{T}$.

2) Let $d_{G}(i, j)$ denote the cost of the shortest path from node $i$ to node $j$ in network $\mathbf{G}$.

3) Let $\pi_{T}(i)$ denote the parent of node $i$ on tree $T, \pi_{T}^{M C}(i)$, the parent MC node of node $i$ on tree $\mathbf{T}$, and $M C^{T}$, the set of MC nodes on tree $\mathbf{T}$.

\section{B. Given}

A sparse-splitting WDM network represented by $\mathrm{G}=(\mathrm{V}$, $\mathrm{E}, \mathrm{c}, \mathrm{M}$ ), where $\mathrm{V}$ is the set of nodes, $\mathrm{E}$ is the set of bidirectional fiber links, $c: E \rightarrow R$ is a channel cost function, $\mathrm{M}$ is the set of MC nodes where $M \subset V$. A non-empty set $r=(s, D) \subset M$ represents the set of nodes, including MI and MC nodes, for a multicast session (s,D). All the destinations can be connected to the tree through the MC and MI nodes.

\section{Output}

We attempt to find a light-tree $\mathbf{T}$ in which there exists a light-path between $(s, d)$ where $d \in D$, and $\mathrm{WC}(\mathrm{T})$ is minimized. In the route, the light splitting constraint must be satisfied, namely, nodes not included in $\mathrm{M}$ cannot split signals. T may use multiple wavelengths on the same link if necessary, but only one transmitter is allowed on $\mathbf{T}$. Assume that there are always free wavelength channels available on all links to construct a light-tree. 


\section{Definition}

An $\mathrm{MC}$ network $\mathbf{M}_{\mathbf{G}}$ of $\mathbf{G}$ is a network whose vertex set, denoted by $W$, consists of the set of MC nodes of $\mathbf{G}$. $d_{G}(i, j)=d_{M_{G}}(i, j)$ for all $i, j \in W$.

\section{E. Lemma 1}

Let $T_{G}^{o p t}(r)$ denote a solution to the minimum wavelength channel cost $\mathrm{MC}$-tree problem in $\mathbf{G}$.

$$
W C_{T_{g}^{o p t}}\left(i, \pi_{T_{G}^{o p t}}^{M C}(i)\right)=d_{G}\left(i, \pi_{T_{G}^{o p t}}^{M C}(i)\right)
$$

for each MC node $i \in M C_{G}^{T_{G}^{o p t}}$.

Proof: If $W C_{T_{G}^{o p t}}\left(i, \pi_{T_{G}^{o p t}}^{M C}(i)\right)>d_{G}\left(i, \pi_{T_{G}^{o p t}}^{M C}(i)\right)$ , we can always replace the original path from $i$ to $\pi_{T}^{M C}(i)$ by this shortest path, resulting in a new lighttree with a smaller cost. Thus, $W C_{T_{G}^{o p t}}\left(i, \pi_{T_{G}^{o p t}}^{M C}(i)\right) \leq$ $d_{G}\left(i, \pi_{T_{G}^{o p t}}^{M C}(i)\right)$. On the other hand, since $d_{G}\left(i, \pi_{T_{G}^{o p t}}^{M C}(i)\right)$ is the cost of the minimum-cost path between $i$ and $\pi_{T_{G}^{o p t}}^{M C}(i), W C_{T_{G}^{o p t}}\left(i, \pi_{T_{G}^{o p t}}^{M C}(i)\right) \geq d_{G}\left(i, \pi_{T_{G}^{o p t}}^{M C}(i)\right)$. Thus, $W C_{T_{G}^{o p t}}\left(i, \pi_{T_{G}^{o p t}}^{M C}(i)\right)=d_{G}\left(i, \pi_{T_{G}^{o p t}}^{M C}(i)\right)$.

Under the sparse-splitting constraint, only MC nodes can serve as the branching nodes to split signals into multiple wavelength channels. Thus, for each destination $d$ of $D$, there exists a light-path, which is a sub-tree of $T_{G}^{o p t}(r)$, connecting $d$ and some on-tree MC nodes. Therefore, $T_{G}^{o p t}(r)$ can be viewed as a set of light-paths connecting the destination to another on-tree MC node, as depicted in Fig. 18. The shaded squares represent the MC nodes that are included in the multicast session. The resulting light-tree can be viewed as six independent light-paths interconnecting MC nodes. This observation leads to the following Lemma.

\section{F. Lemma 2}

Let $T_{G}^{o p t}(r)$ denote a solution to the minimum wavelength channel cost MC-tree problem in $\mathbf{G}$.

$$
W C\left(T_{G}^{o p t}(r)\right)=\sum_{i \in M C_{G}^{T_{G}^{o p t}}} W C\left(i, \pi_{T_{G}^{o p t}}^{M C}(i)\right)
$$

for each MC node $i \in M C_{G}^{T_{G}^{o p t}}$.

\section{G. Theorem 1}

Solving an instance of the minimum wavelength channel cost MC-tree problem for multicast request $r$ in $\mathbf{G}$ is equivalent to solving an instance of the Steiner tree problem for $r$ in the corresponding $\mathbf{M C}$ network $\mathbf{M}_{\mathbf{G}}$. This is a direct consequence of the following Lemma.

\section{H. Lemma 3}

Let $T_{M_{G}}(r)$ denote a Steiner tree in $\mathbf{M}_{\mathbf{G}}$ for multicast request $r .\left|T_{M_{G}}(r)\right|=W C\left(T_{G}^{o p t}(r)\right)$.

Proof: Suppose that a minimum cost tree $T_{G}^{o p t}(N)$ is given, and let $T_{M_{G}}(r)$ denote a Steiner tree for $r$ in $\mathbf{M}_{\mathbf{G}}$. Based on Lemma 1 and Lemma $2, W C\left(T_{G}^{o p t}(r)\right)=$ $\sum_{i \in M C^{T_{G}^{o p t}}} W C\left(i, \pi_{T_{G}^{o p t}}^{M C}(i)\right)=\sum_{i \in M C_{G}^{T_{G}^{o p t}}} d_{G}\left(i, \pi_{T_{G}^{o p t}}^{M C}(i)\right)$ is minimal in building the light-tree connecting all members in $r$. From the definition of the MC network, an equal cost path $p_{i}$ between $i$ and $\pi_{M C}(i)$ can always be found in $\mathbf{M}_{\mathbf{G}}$. By including all edges of $p_{i}$ between $i$ and $\pi_{T_{G}^{o p t}}^{M C}(i)$ in $\mathbf{M}_{\mathbf{G}}$ for $i \in M C^{T_{G}^{o p t}}$, we can construct a tree $T^{\prime}$ spanning all members included in $r$ with $\left|T^{\prime}\right| \leq W C\left(T_{G}^{o p t}(r)\right)$. Thus,

$$
\left|T_{M_{G}}(r)\right| \leq\left|T^{\prime}\right| \leq W C\left(T_{G}^{o p t}(r)\right) .
$$

On the other hand, suppose that a Steiner minimal tree $T_{M_{G}}(r)$ is given. For each link $(i, j)$ in $T_{M_{G}}(r)$, a light-path $L_{j}^{i}$ connecting node $i$ to $j$ in $\mathbf{G}$ with $W C\left(L_{j}^{i}\right)=c(i, j)$ can always be found. By including every light-path $L_{j}^{i}$ generated from each $\operatorname{link}(i, j)$ in $T_{M_{G}}(r)$, we can construct a lighttree $T^{\prime \prime}$ with $W C\left(T^{\prime \prime}\right)=\left|T_{M_{G}}(r)\right|$. Thus, $\left|T_{M_{G}}(r)\right|=$ $W C\left(T^{\prime \prime}\right) \geq W C\left(T_{G}^{o p t}(r)\right)$.

Therefore, $\left|T_{M_{G}}(r)\right|=W C\left(T_{G}^{o p t}(r)\right)$.

\section{REFERENCES}

[1] L. H. Sahasrabuddhe and B. Mukherjee, "Light-trees: optical multicasting for improved performance in wavelength-routed networks," IEEE Commun. Mag., vol. 37, pp. 67-73, Feb. 1999.

[2] X. Zhang et al., "Constrained multicast routing in WDM network with sparse light splitting," IEEE J. Lightwave Technol., vol. 18, no. 12, Dec. 2000.

[3] S. Yan et al., "Route optimization of multicast sessions in sparse lightsplitting optical networks," in Proc. IEEE GLOBECOM 2001.

[4] W.-Y. Tseng et al., "All-optical multicasting on wavelength-routed WDM networks with partial replication," in Proc. IEEE ICC '01, Helsinki, Finland, June 2001.

[5] N. Sreenath, "Virtual source based multicast routing in WDM networks with sparse-light splitting," in Proc. IEEE High Performance Switching and Routing 2001.

[6] F. Hwang and D. Richards, "Steiner tree problems," Networks, vol. 22, pp. $55-89,1992$

[7] H. Takahashi and A. Matsuyama, "An approximate solution for the Steiner problem in graphs," Math. Japonica, vol. 24, no. 6, pp. 573577.

[8] L. Kou, G. Markowsky, and L. Berman, "A fast algorithm for Steiner trees," Acta Informatica, pp. 141-145, 1981.

[9] M. Smith and P. Winter, "Path-distance heuristics for the Steiner problem in undirected networks," Algorithmica, vol. 7, no. 2-3, pp. 309-327, 1992

[10] Wang and J. Hou, "Multicast routing and its QoS extension problems, algorithms, and protocols," IEEE Network, pp. 22-36, Jan. 2000.

[11] W. S. Hu and Q. J. Zeng, "Multicasting optical cross connects employing splitter-and-delivery switch," IEEE Photon. Technol. Lett., vol. 10, pp. 970-972, July 1998.

[12] B. Waxman, "Routing of multipoint connections," IEEE J. Sel. Areas Commun., vol. 6, no. 9, pp. 1617-1622, Dec. 1988.

[13] K. Calvert and E. Zegura, "GT internetwork topology models (GTITM)," available at: http://www.cc.gatech.edu/fac/Ellen.Zegura/gt-itm

[14] A. Shaikh and K. Shin, "Destination-driven routing for low-cost multicast," IEEE J. Sel. Areas Commun., vol. 15 no. 3, pp. 373 -381, April 1997.

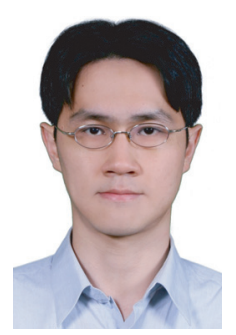

Cheng-Yu Hsieh received the B.S. and M.S. degrees in Electronic Engineering from the Department of Electrical Engineering, National Taiwan University in 2000 and 2002, respectively. He is currently an engineer in Media-Tek Inc. in Taiwan. Her research interest is in wireless and multimedia communications. 


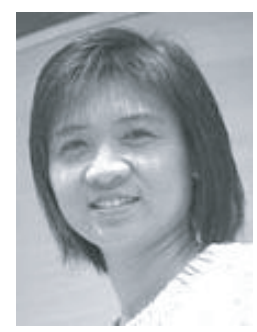

Wanjiun Liao received the BS and MS degrees from National Chiao Tung University, Taiwan, in 1990 and 1992, respectively, and the Ph.D. degree in Electrical Engineering from the University of Southern California, Los Angeles, California, USA, in 1997. She joined the Department of Electrical Engineering, National Taiwan University (NTU), Taipei, Taiwan, as an Assistant Professor in 1997. Since August 2005, she has been a full professor. Her research interests include wireless networks, works. multimedia networks, and broadband access net-

Dr. Liao is currently an Associate Editor of IEEE Transactions on Wireless Communications and IEEE Transactions on Multimedia. She served as the
Technical Program Committee (TPC) chairs/co-chairs of many international conferences, including the Tutorial Co-Chair of IEEE INFOCOM 2004, the Technical Program Vice Chair of IEEE Globecom 2005 Symposium on Autonomous Networks, and the Technical Program Co-Chair of IEEE Globecom 2007 General Symposium. Dr. Liao has received many research awards. Papers she co-authored with her students received the Best Student Paper Award at the First IEEE International Conferences on Multimedia and Expo (ICME) in 2000, and the Best Paper Award at the First IEEE International Conferences on Communications, Circuits and Systems (ICCCAS) in 2002. Dr. Liao was the recipient of K. T. Li Young Researcher Award honored by ACM in 2003, and the recipient of Distinguished Research Award from National Science Council in Taiwan in 2006. She is a Senior member of IEEE. 